collection suffisante de souches entraînées à résister à des concentrations variées des divers antiseptiques et antibiotiques susceptibles d'être rencontrés dans le lait. Nous avons créé une telle collection. Des laboratoires spécialisés pourraient en créer de semblables, les entretenir, et les distribuer au fur et à mesure des besoins.

Cette technique des.souches résistantes peut être étendue à l'étude de nombreux autres problèmes: dépistage d'autres facteurs d'inhibition (pour autant qu'ils soient isolables, même s'ils sont inconnus) création de souches mixtes polyrésistantes pour l'industrie laitière et fromagère, application des mêmes principes à la recherche des antiseptiques ou des antibiotiques dans d'autres produits que le lait et à la lutte contre certains accidents de fabrication d'origine bactériologique dans d'autres industries.

\title{
CONTRIBUTION A L'ÉTUDE DE LA COMPOSITION DU LAIT
}

\section{Anhydrase carbonique, rhodanase et phosphomonoestérase alcaline dans les laits de vache, de chèvre et de brebis (1) \\ par \\ Cristino GARCIA ALFONSO \\ et \\ Enrìue CASTELLA BERTRÁN}

La composition du lait est actuellement un problème résolu, en particulier pour ce qui concerne les parties macropondérales. Parmi les éléments biologiques, les vitamines sont graduellement déterminées l'une après l'autre dans les divers laits.

En ce qui concerne les ferments, nous en connaissons parfaitement un petit groupe, qui trouve son application dans les services de contrôle pour vérifier la température à laquelle a été soumis un lait déterminé. Les autres enzymes ont été presque oubliées, manquant d'utilité pratique immédiate. Elles ne semblent pas constituer, comme les vitamines, un facteur de grande importance dans la nutrition des êtres qui consomment du lait. Leur connaissance présente seulement un simpleintérêt scientifique, en tant que donnée à ajouter à celles déjà connues sur la composition du lait, ou en tant qu'indice de la circulation et de l'excrétion des enzymes organiques.

Nous apportons dans ce travail une série de données sur la carbonicoanhydrase, la rhodanase et la phosphomonoestérase

(1) Annales dela Faculté de Médecine Vétérinaire del'Université de Madrid, 1952, vol. IV, fasc. 1, I. 
alcaline. Dans une autre communication publiée dans ce même volume, nous donnons un recueil des valeurs au sujet de la phosphomonoestérase acide, toujours dans les laits de chèvre, de vache et de brebis.

Les phosphomonoestérases ont été beaucoup étudiées dans le lait de vache, en particulier la phosphomonoestérase alcaline, Dans le lait de chèvre également, on a effectué quelques recherches, mais très rarement dans le lait de brebis. Nous insistons sur l'intérêt du sujet, puisque nous employons une technique beaucoup plus sensible que celles qui sont utilisées habituellement dans les services de contrôle, mais en outre, pour citer des valeurs quantitatives, et non simplement qualitatives, comme le font couramment les auteurs qui effectuent des études sur le lait.

En ce qui concerne la carbonicoanhydrase, nous n'avons rencontré, dans l'abondante bibliographie que nous avons consultée, aucune mention de ses valeurs dans le lait. Nous remplissons donc, pour ainsi dire, un vide. Nous faisons de même pour la rhodanase. Aucun des auteurs qui ont étudié ce ferment ne l'a cherché dans le lait. Dans une publication antérieure [1], où nous avions étudié le ferment dans les animaux domestiques, en donnant des valeurs globales et comparatives, nous attribuions déjà au lait de vache une teneur pratiquement nulle, puisque les quelques dixièmes d'unité parfois constatés n'ont pas d'importance, comparés aux centaines d'unités présentes dans les autres organes ou humeurs.

Nous décrirons ci-après les trois ferments, séparément, et, pour chaque enzyme isolément, selon les espèces.

\section{Carbonicoanhydrase}

Ce ferment, appelé aussi anhydrase carbonique, a pour fonction la déshydratation de l'acide carbonique et l'hydratation de l'anhy. dride carbonique. Il abonde extraordinairement dans les érythrocytes et la muqueuse gastrique. Nous l'avons étudié dans un travail antérieur [2] sur la poule, l'œuf et l'embryon pendant l'incubation, et actuellement nous l'évaluons dans les matières organiques de diverses espèces domestiques ou de laboratoire.

Pour sa détermination, nous avons suivi la méthode de FLoRKIN [3], basée sur la déshydratation de l'acide carbonique et l'appréciation de la variation du $p \mathrm{H}$ au moyen du rouge de phénol.

Dans le tableau I, nous exposons les résultats donnés par les échantillons de lait analysés, douze pour chaque espèce, après étude des laits de vache, de chèvre et de brebis, obtenus d'animaux de races, âges, et conditions différents, parmi ceux qui approvisionnent habituellement en lait la ville de Madrid. 
Dans la méthode que nous employons, on calcule le temps nécessaire pour une variation de $p \mathrm{H}$ suffisante pour faire changer de couleur le réactif indicateur. Le temps employé et la quantité de ferment sont en relation inverse. Les produits riches en ferment nécessitent moins de temps que les autres, plus pauvres en enzyme. Le temps est exprimé en secondes.

TABLEAU I

LA GARBONICOANHYDRASE DANS LE LAIT

\begin{tabular}{c|c|c|c|c|c|c|c|c|c|c|c|c|c}
\hline \hline Animal no & 1 & 2 & 3 & 4 & 5 & 6 & 7 & 8 & 9 & 10 & I I & 12 & $\begin{array}{c}\text { Moy- } \\
\text { ennè }\end{array}$ \\
\hline Espèce . & & & & & & & & & & & & & \\
Vache ... & 10 & 10 & 10 & 1.0 & 16 & 16 & 12 & 22 & 25 & 12 & 10 & 15 & 14 \\
Brebis.... & 25 & 25 & 25 & 15 & 12 & 12 & 25 & 20 & 15 & 15 & 22 & 23 & 18 \\
Chèvre ... & 20 & 20 & 12 & 17 & 15 & 20 & 20 & 16 & 13 & 10 & 20 & 15 & 16,5 \\
\hline \hline
\end{tabular}

Temps du virage exprimé en secondes.

A l'examen du tableau I, nous pouvons apprécier des détails du plus grand intérêt. En premier lieu, nous voyons que les chiffres moyens et ceux donnés par les différents échantillons sont assez semblables dans les trois laits. Dans le lait d'une même espèce, il y a une grande diversité de l'un à l'autre des échantillons. Le Iait le plus riche, donc celui qui demande le moins de temps pour le virage, est celui de vache, avec une moyenne de 14 secondes, bien que les oscillations soient plus grandes, de 10 à 25 secondes. Le lait de chèvre demande en moyenne 16 secondes et demie pour donner lieu à un changement de coloration, avec des minima de 12 et des maxima de 25 secondes. Le lait de brebis est le plus pauvre en anhydrase carbonique, mais nécessite environ 18 secondes en moyenne pour le virage, avec des oscillations entre 12 et 25 secondes également.

Ces valeurs doivent être considérées exclusivement au sens qualitatif et comme comparaison des trois ferments entre eux. Les méthodes qui existent pour la détermination de la carbonicoanhydrase sont encore complexes et imprécises, ce qui, joint à la grande ressemblance des trois laits quant à leur richesse en ferment, empêche leur utilisation pour des fins pratiques de recherche des températures auxquelles on a soumis le lait, ou pour la différenciation des laits entre eux.

En comparaison avec les autres matières organiques, le lait est pauvre en carbonicoanhydrase. Les temps obtenus s'assimilent à eeux donnés par les macérations d'organes pauvres en cette enzyme, 
macérations diluées à $1 / 10^{\mathrm{e}}$, ce qui signifie que le lait contient dix fois moins de ferment que le contenu de ces organes. Le sang principalement, est beaucoup plus riche en cette enzyme que le lait.

\section{Rhodanase}

La rhodanase favorise la conversion du cyanure, hautement toxique, en sulfocyanures presque inoffensifs.

Antériearement, nous avons étudié ce ferment dans la plupart des organes des animaux domestiques et de laboratoire. Nous renvoyons à ces publications le lecteur qui désirerait de plus amples informations sur le ferment. Pour sa détermination, nous avons employé la méthode de CosBy et SUMNer [4], en exprimant les résultats en unités propres. Nous exposons les données obtenues dans les tableaux II, III et IV. Le premier correspond au lait de vache, dont on a étudié vingt échantillons appartenant à autant d'animaux.

TABLEAU II

LA RHODANASE DANS LE LAIT DE VAGHE

\begin{tabular}{|c|c|c|c|c|c|}
\hline $\begin{array}{l}\text { Vache } \\
\text { no }\end{array}$ & \multicolumn{2}{|c|}{$\begin{array}{l}\text { Unités (par } \\
\text { centimetre } \\
\text { cube) }\end{array}$} & $\begin{array}{c}\text { Vache } \\
\text { no }\end{array}$ & \multicolumn{2}{|c|}{$\begin{array}{c}\text { Unités (par } \\
\text { centimètre } \\
\text { cube) }\end{array}$} \\
\hline $1 \ldots$ & $\ldots$ & 0,35 & $11 \ldots$ & $\ldots$ & 0,54 \\
\hline $2 \ldots$ & $\ldots$ & 0 & $12 \ldots$ & $\ldots$ & 0 \\
\hline $3 \ldots$ & $\ldots$ & 0,06 & $13 \ldots$ & $\ldots$ & 0,46 \\
\hline $4 \ldots$ & $\ldots$ & $0^{\prime}$ & $14 \ldots$ & $\ldots$ & 0 \\
\hline $5 .$. & $\ldots$ & 1,07 & $15 \ldots$ & $\ldots$ & 1,7 \\
\hline $6 \ldots$ & $\ldots$ & 0 & $16 \ldots$ & $\cdots$ & 0,64 \\
\hline $7 \ldots$ & $\ldots$ & 0 & $17 \ldots$ & $\ldots$ & 0 \\
\hline 8.:. & $\ldots$ & 0 & $18 \ldots$ & $\cdots$ & 0,12 \\
\hline $9 \ldots$ & $\ldots$ & 1 & $19 \ldots$ & $\ldots$ & 0 \\
\hline $10 \ldots$ & $\ldots$ & 0 & $20 \ldots$ & $\ldots$ & 0,5 \\
\hline
\end{tabular}

Monenne : 0,34 .

En général, le lait a peu de rhodanase, mais le lait de vache est le plus pauvre parmi les divers laits. Beaucoup d'échantillons ne permettent même pas d'apprécier des traces du ferment, et les plus riches en possèdent seulement une unité par centimètre cube de lait. La moyenne des vingt échantillons fut de 0,34 unité par centimètre cube, chiffre ridicule en comparaison des 1.000 unités par gramme que possèdent beaucoup d'organes.

De même, le lait de chèvre a peu de ferment, mais cependant sensiblement plus que le lait de vache. La moyenne, sur quinze échantillons provenant d'autant d'animaux, a été de 1,24 unités 
par centimètre cube, avec des minima de 0 (très rares) et des maxima d'environ 3 unités par centimètre cube.

TABLEAU III

LA RHODANASE DANS LE LAIT DE GHËVRE
TABLEAU IV

LA RHODANASE DANS LE LAIT DE BREBIS

\begin{tabular}{|c|c|c|c|}
\hline $\begin{array}{c}\text { Chèvre } \\
\text { na }\end{array}$ & $\begin{array}{l}\text { Tnités (par } \\
\text { entimètre } \\
\text { cube) }\end{array}$ & $\begin{array}{c}\text { Brebis } \\
n^{\circ}\end{array}$ & $\begin{array}{l}\text { (par } \\
\text { nètrie } \\
\text { e) }\end{array}$ \\
\hline $1 \ldots \ldots \ldots \ldots \ldots \ldots \ldots$ & . & $1 \ldots \ldots \ldots \ldots \ldots \ldots \ldots$ & 3,76 \\
\hline $2 \ldots \ldots \ldots \ldots \ldots \ldots \ldots$ & 0,8 & $2 \ldots \ldots \ldots \ldots \ldots \ldots \ldots \ldots$ & 1,18 \\
\hline $3 \ldots \ldots \ldots \ldots \ldots \ldots \ldots$ & 0,46 & $3 \ldots, \ldots \ldots \ldots \ldots \ldots \ldots$ & 1,2 \\
\hline $4 \ldots \ldots \ldots \ldots \ldots \ldots \ldots$ & 0 & $4 \ldots \ldots \ldots \ldots \ldots \ldots \ldots \ldots$ & 3,36 \\
\hline $5 \ldots \ldots \ldots \ldots \ldots \ldots \ldots \ldots$ & 2,74 & $5, \ldots \ldots \ldots \ldots \ldots \ldots \ldots$ & 1,2 \\
\hline $6 \ldots \ldots \ldots \ldots \ldots \ldots \ldots$ & 2,07 & $6, \ldots \ldots \ldots \ldots \ldots \ldots \ldots$ & 2,4 \\
\hline $7 \ldots \ldots \ldots \ldots \ldots \ldots \ldots$ & 2,07 & $7 \ldots \ldots \ldots \ldots \ldots \ldots \ldots$ & 1,5 \\
\hline $8 \ldots \ldots \ldots \ldots \ldots \ldots \ldots$ & 2,98 & $8 \ldots \ldots \ldots \ldots \ldots \ldots \ldots$ & 1 \\
\hline $9 \ldots \ldots \ldots \ldots \ldots \ldots$ & 0,8 & $9 \ldots \ldots \ldots \ldots \ldots \ldots$ & 3,2 \\
\hline $10, \ldots \ldots \ldots \ldots \ldots \ldots \ldots$ & 1,5 & $10 \ldots \ldots \ldots \ldots \ldots \ldots$ & 2,3 \\
\hline $11 \ldots \ldots \ldots \ldots \ldots \ldots \ldots \ldots$ & 0,5 & $11 \ldots \ldots \ldots \ldots \ldots \ldots \ldots$ & 1,3 \\
\hline $12 \ldots \ldots \ldots \ldots \ldots \ldots \ldots$ & 1 & $12 \ldots \ldots \ldots \ldots \ldots \ldots \ldots$ & 1,7 \\
\hline $13 \ldots \ldots \ldots \ldots \ldots \ldots \ldots$ & 1,7 & $13 \ldots \ldots \ldots \ldots \ldots \ldots \ldots \ldots$ & 1,1 \\
\hline $14 \ldots \ldots \ldots \ldots \ldots \ldots \ldots$ & 2 & $14 \ldots \ldots \ldots \ldots \ldots \ldots \ldots$ & 3,5 \\
\hline $15 \ldots \ldots \ldots \ldots \ldots \ldots \ldots$ & . & $15 \ldots \ldots \ldots \ldots \ldots \ldots \ldots \ldots$ & 1 \\
\hline
\end{tabular}

Le tableau IV donne les résultats obtenus en analysant le lait de quinze brebis. Le lait de brebis est le plus riche des trois laits. La moyenne est de 2 unités par centimètre cube et les maxima sont approximativement de 4 unités. Les valeurs minima ne sont pas inférieures à l'unité.

Le lait de brebis présente aussi une autre caractéristique, assurément dérivée de sa plus grande richesse en rhodanase. Il possède notablement plus de rhodanate (aussi appelé sulfocyanure ou thiocyanate) que les laits de chèvre et de vache, le double environ des valeurs trouvées dans ces derniers laits.

La petite quantité de rhodanase présente dans le lait et sa détermination délicate empêchent de pouvoir employer le ferment comme élément d'appréciation des températures subies par un lait. Bien que la différence soit notable entre la quantité de rhodanase que possèdent les laits de chèvre, de brebis et de vache, nous ne pouvons non plus nous fixer sur ce détail pour différencier ces laits, car cette quantité de ferment, trop minime, ne permet pas des déterminations capables de fournir des résultats concluants. 


\section{Phosphomonoestérase alcaline}

Comme nous l'avons déjà indiqué antérieurement, la phosphomonoestérase alcaline du lait a fait couler beaucoup d'encre. Dans cette partie du travail, nous ne prétendons pas découvrir des faits nouveaux, mais simplement donner connaissance de chiffres obtenus en employant notre méthode [5], très exacte en vérité, sur des animaux d'Espagne, et notamment de Madrid, et faire en même temps une étude comparative des laits de vache, de chèvre et de brebis. Pour ces deux derniers, les données concernant la phosphomonoestérase alcaline sont encore assez obscures, en particulier pour le lait de brebis.

TABLEAU $V$

PHOSPHOMONOESTÉRASE ALGALINE DANS L八 LAIT DE VACHE

\begin{tabular}{|c|c|c|c|c|c|c|c|c|}
\hline Vaches no $\ldots .$. & 1 & 2 & 3 & 4 & 5 & 6 & 7 & 8 \\
\hline Echantillons $n^{\circ} \ldots$ & $1-2-3$ & $1-2-3$ & ] $-2-3$ & $1-2-3$ & $1-2-3$ & $1-2-3$ & $1-2-3$ & $1-2-3$ \\
\hline \multirow{3}{*}{$\begin{array}{l}\text { Unités Bodansky.. } \\
\left.\text { (par } 100 \mathrm{~cm}^{3}\right)\end{array}$} & 8,8 & 0 & 7,5 & 3,5 & 6,7 & 0,7 & 5,34 & 12,8 \\
\hline & 8,8 & 0 & 0 & 2,9 & 5,9 & 1,28 & 12,54 & 13,6 \\
\hline & 7,5 & 0,5 & 3,2 & 4,1 & 4,8 & 1 & 8,55 & 11,7 \\
\hline Vaches n ${ }^{0} \ldots \ldots$ & 9 & 10 & 11 & 12 & 13 & 14 & 15 & \\
\hline Echantillons n ${ }^{\circ} .$. & $1-2-3$ & $1-2-3$ & $1-2-3$ & $1-2-3$ & $1-2-3$ & $1-2-3$ & $1-2-3$ & \\
\hline \multirow{3}{*}{$\begin{array}{l}\text { Unités Bodansky.. } \\
\qquad\left(\text { par } 100 \mathrm{~cm}^{3}\right)\end{array}$} & 0 & 0 & 8,8 & 2,6 & 22 & 0 & 13 & \\
\hline & 0 & 0 & 11 & 10,3 & 14,8 & 0 & 12,5 & \\
\hline & 1,2 & 0 & 10,5 & 5,8 & 17 & 0 & 7,3 & \\
\hline
\end{tabular}

Moyenne: 6 .

Les quarante-cinq analyses effectuées avec les laits provenant de quinze vaches sont réunies dans le tableau $V$. Ces analyses permettent d'émettre les affirmations suivantes : le lait de vache possède assez de phosphomonoestérase alcaline, mais il n'est pas un produit riche en ce ferment, comme on pourrait le supposer, étant donné le fait que l'on parle beaucoup de cette enzyme dans ce liquide. Les chiffres sont bien plus limités. Beaucoup de matières organiques possèdent des valeurs immensément plus élevées ; tout d'abord, nous avons toujours travaillé sur des laits frais, récemment " tirés ", sans modification, ni traitement par la chaleur, donc avec toute leur richesse enzymatique intégrale. Ce fait étant bien établi, nous considérons que les méthodes de détermination de la phosphatase utilisées par les services d'inspection des laits pour le contrôle de la pasteurisation ou la détection du lait bouilli doivent être suffisamment correctes et employées par un personnel compétent, sous peine d'erreurs nombreuses. 
La moyenne des quarante-cinq échantillons s'élève à 6 unités Bodansky par $100 \mathrm{~cm}^{3}$ de lait, avec un maximum de 22 unités dans l'échantillon $n^{0} 1$ de la vache $n^{0} 13$, mais en général, les maxima ne dépassent pas 12 à 14 unités. En revanche, il y a lieu d'insister sur le fait que beaucoup de vaches ne possèdent pas de phosphomonoestérase alcaline (vulgairement phosphatase) dans leur lait, de sorte que nous pourrions facilement croire bouilli un lait parfaitement frais. En d'autres termes, l'épreuve de la phosphatase, pour déterminer si un lait a été bouilli, a une valeur pour des mélanges de laits de plusieurs vaches, mais non pour le lait provenant d'un seul animal. Dans un mélange de laits frais, principalement, il est presque impossible que le ferment soit absent.

Le lait de chèvre possède plus d'enzyme que le lait de vache. Comme on peut l'apprécier par l'examen du tableau VI, on obtient une moyenne de 10,5 unités Bodansky par $100 \mathrm{~cm}^{3}$, après avoir analysé le lait de quinze animaux différents. Les résultats sont plus eonstants que dans le lait de vache. Le maximum ne dépasse pas 14 unités, et le minimum ne descend pas au-dessous de 9 unités. Comme l'avaient affirmé déjà certains auteurs en contradiction avec l'opinion de quelques autres, l'épreuve de la phosphatase a autant de valeur pour le lait de chèvre que pour le lait de vache. Nous pouvons dire encore plus, puisque par la plus grande quantité

TABLEAU VI

LA PHOSPHOMONOESTÉRASE ALCALINE DANS LE LAIT DE GHËVRE

\begin{tabular}{|c|c|}
\hline $\begin{array}{c}\text { Chèvre } \\
n^{\circ}\end{array}$ & $\begin{array}{l}\text { nités } \\
\text { lansky } \\
100 \mathrm{~cm}^{3} \text { ) }\end{array}$ \\
\hline $1 \ldots \ldots \ldots \ldots \ldots$ & 10,1 \\
\hline $2 \ldots \ldots \ldots \ldots \ldots \ldots$ & 12,5 \\
\hline $3 \ldots \ldots \ldots \ldots \ldots \ldots \ldots \ldots \ldots \ldots \ldots \ldots$ & 11,2 \\
\hline $4 \ldots \ldots \ldots \ldots \ldots$ & 10,1 \\
\hline 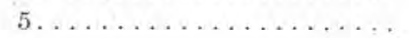 & 12,25 \\
\hline 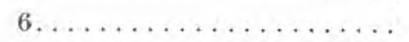 & 13,66 \\
\hline 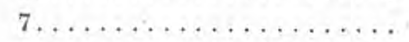 & 9,2 \\
\hline $8 \ldots \ldots \ldots \ldots \ldots \ldots \ldots \ldots \ldots \ldots \ldots$ & 9,5 \\
\hline 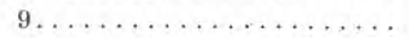 & 11,7 \\
\hline 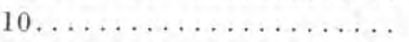 & 9,5 \\
\hline $11 \ldots \ldots \ldots \ldots \ldots \ldots$ & 13 \\
\hline 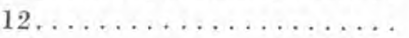 & 12,5 \\
\hline $13 \ldots \ldots \ldots \ldots \ldots \ldots \ldots \ldots \ldots \ldots$ & 10 \\
\hline $14 \ldots \ldots \ldots \ldots \ldots \ldots \ldots \ldots \ldots \ldots$ & 11,6 \\
\hline
\end{tabular}

Moyenne: 10,5 .
TABLEAU VII

LA PHOSPHOMONOESTÉRASE ALGALINE DANS LE LAIT DE BREBIS

$\begin{array}{cc}\text { Brebis } & \text { Unités } \\ \mathrm{n}^{\circ} & \text { Bodansky } \\ & \left(\text { par } 100 \mathrm{~cm}^{3}\right)\end{array}$

\begin{tabular}{|c|c|}
\hline $1 \ldots \ldots \ldots \ldots \ldots \ldots \ldots$ & 11 \\
\hline $2 \ldots \ldots \ldots \ldots \ldots \ldots \ldots \ldots \ldots$ & 12,6 \\
\hline $3 \ldots \ldots \ldots \ldots \ldots \ldots \ldots$ & 16,6 \\
\hline $4 \ldots \ldots \ldots \ldots \ldots \ldots \ldots$ & 4,4 \\
\hline $5 \ldots \ldots \ldots \ldots \ldots \ldots \ldots$ & 18,4 \\
\hline $6 \ldots \ldots \ldots \ldots \ldots \ldots$ & 18,4 \\
\hline $7 \ldots \ldots \ldots \ldots \ldots \ldots \ldots \ldots \ldots \ldots$ & 30,6 \\
\hline 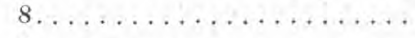 & 25,2 \\
\hline $9 \ldots \ldots \ldots \ldots \ldots \ldots \ldots$ & 19 \\
\hline 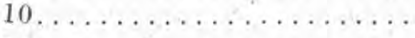 & 17 \\
\hline 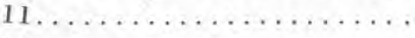 & 17,2 \\
\hline $2 \ldots \ldots \ldots \ldots \ldots \ldots \ldots \ldots$ & 11,2 \\
\hline 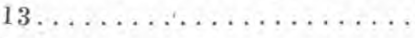 & 6 \\
\hline $4 \ldots \ldots \ldots \ldots \ldots \ldots \ldots \ldots$ & 7,5 \\
\hline $5 \ldots \ldots \ldots \ldots \ldots \ldots \ldots \ldots \ldots \ldots \ldots \ldots$ & 12,5 \\
\hline
\end{tabular}

Moyenne: 15 . 
de ferment et le fait qu'on n'observe pas de laits individuels man: quant d'enzyme, la ceertitude qu'un lait est bouilli est plus grande, pour le chimiste qui effectue l'analyse, que s'il s'agit de lait de vache. En outre, nous pouvons tolérer moins d'exactitude dans les méthodes, en raison de cette richesse plus grande par rapport au lait de vache.

Le lait de brebis est, des trois laits étudiés, celui qui possède les plus grandes quantités de ferment. La moyenne des laits de quinze brebis fut de 15 unités Bodansky par $100 \mathrm{~cm}^{3}$ de lait, moyenne relativement plus élevée que celle du lait de chèvre et très supérieure à celle du lait de vache. La dispersion des résultats est plus manifeste que dans le tablean correspondant au lait de chèvre. Le minimum fut de 4,4 unités pour la brebis no 4 et le maximum, de 30,6 unités pour la brebis no 7 (voir tableau VII).

L'épreuve des phosphatases, pour les raisons exposées en parlant du lait de chèvre, a une valeur équivalente ou supérieure dans le lait de brebis à celle qui existe dans le lait de vache, par l'absence de lait manquant de ferment et par la moyenne plus élevée.

Malgré les différences notables que présente le ferment dans les trois espèces, nous ne pouvons l'utiliser comme élément d'appréciation pour différencier les trois laits entre eux ou pour détecter le lait de brebis dans le lait de vache, par exemple, puisque, comme nous l'avons déjà écrit dans un autre article récent dédié à la phosphomonoestérase acide des trois laits, les ferments n'ont pas la stabilité des chlorures ou du lactose, par exemple, mais qu'ils sont des composés vivants suprêmement affectés par les facteurs ambiants : température, vieillissement, dilution, etc., qui donnent des résultats très variés et enfin des conclusions basées sur ces résultats, qui sont toujours très aventureuses.

Nous remercions MM. Eduardo OLALLA Herrero et Victorino Vicente Martin, élèves internes au Laboratoire Annexe de la Chaire de Pharmacologie de la Faculté de Médecine Vétérinaire de Madrid, pour l'aide manuelle prêtée au cours de cette étude.

\section{BIBLIOGRAPHIE}

[1] F. Sanz, E. Castella, J. A. Tarazona. An. Fac. Vétérinaria, Madrid, 1950, II, 97.

[2] E. Castella. Tésis doctoral, Madrid, 1952.

[3] Flonkin. Arch. Internat. Physiol., 1934, 40, 283.

[4] E. Cosbi, J. B. Summer. Arch. Biochem., 1945, 7, 457.

[5] E. Castelda. An. Fac. Vétérinaria, Madrid, 1950, II, 50. 